\title{
Protecting Multimedia Authenticity With ICA Vaccination of Digital Bacteria Watermarks
}

\author{
Harold Szu ${ }^{1}$, Steven Noel ${ }^{2}$, Seong-Bin Yim ${ }^{3}$, Jeff Willey ${ }^{4}$, and Joe Landa ${ }^{5}$ \\ ${ }^{1}$ Office of Navel Research, 800 N. Quincy St., Arlington VA 22217-5660 \\ ${ }^{2}$ Center for Secure Information Systems, George Mason University, VA 22030-4444 \\ ${ }^{3}$ Digital Media RF Lab, ECE Dept., The George Washington University, Washington DC 20052 \\ ${ }^{4}$ Code 5344, Naval Research Laboratory, Washington, DC 20375-5336 \\ ${ }^{5}$ BriarTek, Inc., 112 E. Del Ray Ave., Suite A, Alexandria, VA 22301
}

\begin{abstract}
We propose the application of independent component analysis (ICA), via unsupervised neural networks, to authenticity protection for multimedia products. We give an overview of the current state of multimedia authenticity protection, including the requirements of various multimedia applications, current approaches to the problem, and the robustness of the approaches. For watermark security, a covert independentcomponent watermarking signal can serve as a "vaccination" against a dormant digital "bacteria" protecting the multimedia data. Unauthorized removal of the watermark triggers the bacterium, which then responds appropriately against the pirated data. We insure that our digital bacteria meet established requirements for beneficial virus-like programs. Overall, we show how these new approaches contribute to a flexible, robust, and secure system for protecting the authenticity of multimedia products.
\end{abstract}

Keywords-Multimedia watermark, copyright protection, Internet commerce, independent component analysis, unsupervised neural networks.

\section{INTRODUCTION}

Noel and Szu [1] introduced in 2000 the application of independent Component Analyses (ICA) to digital watermarking for multimedia authenticity protection [2]-[4]. The ubiquitous piracy of copyrighted digital music files over the Internet threatens the livelihood of content-providing businesses as well as the creative artists who hold the copyright. Besides the legal and practical issues related to copyright enforcement, several technology factors influence the piracy. The major technology component is the presence of easy to use Internet based peer-to-peer file-sharing technology. Another factor is the compact format and high fidelity of Moving Picture Expert Group-I (MPEG-I) Layer-3 (MP3) digitally encoded music files. The result is that peer-to-peer MP3 encoded music files can be obtained quickly over high-speed network connections.

Many technical strategies have been both proposed and pursued to reduce the piracy problem. One approach is to embed a digital code or watermark in the music itself. The watermark can be chosen to be imperceptible to the listener or deliberately chosen to corrupt the musical audio signal.

The ideal watermarking technique for digital music files has several desirable attributes including (1) compatibility with the popular MP3 format, (2) resistance to watermark removal by the consumer, and (3) complete recovery of the music from the watermark with authorized/restricted computer codes. We explore the efficacy of a novel approach (linear mixing and blind demixing with ICA) for watermarking digital music files to fulfill these goals.

Earlier research on the use of watermarking with ICA concentrated on detection [2][3][5]-[7] and application to imagery $[1][4][8][10][11]$. This report focuses on some of the subtle and practical issues of ICA for watermarking digital music files.

Noel and Szu [1] described two models for watermarking of multimedia products: (1) a standard model and (2) a model for neural net ICA demix enabled media player (Fig. 1 and Fig. 2, respectively). The model in Fig. 1 uses a standard media player; therefore, the hidden watermark is invisible. On the other hand, the model in Fig. 2 uses an ICA demixed media player to recover the hidden watermark when proving copyright. This previous paper points out a critical issue: ICA needs to be able to demix signals that have been subjected to lossy compression. It also shows that ICA neural networks can blindly demix audio data signals with minimal distortion even after the application of lossy wavelet compression.

In this paper, the idea is to nonlinearly mix a watermark audio signal with the music signal, MP3 encode and decode

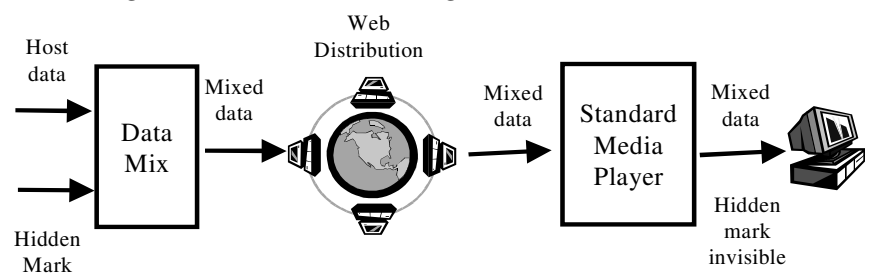

Fig. 1. Standard watermarking model.

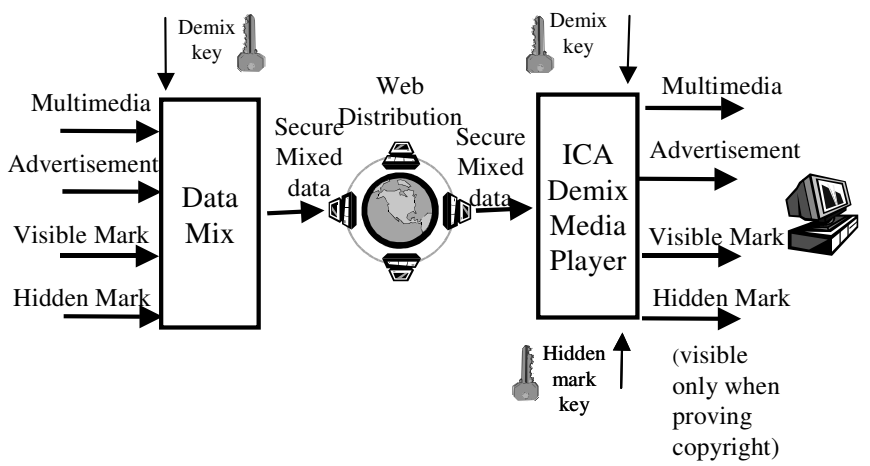

Fig. 2. ICA demix enabled model. 
the resulting signal, then attempt to demix the watermark signal from the music. The novelty of this method introduces difficulty in the removal of the watermark by the unsophisticated consumer. The value of restricted computer codes to restore the music from a watermarked signal will depend in part on the quality of music after blind demixing with ICA [12]-[14][18]-[20]. We measure the watermark contamination of the music after blind demixing with ICA with MP3 encoding.

Excellent progress has been made toward blind demixing via unsupervised neural networks [1]-[4][8][9], an approach known as independent component analysis (ICA) [22][23]. The original signals are modeled as statistically independent signal components, which are then linearly mixed. Here independence is over all orders of statistics, not just $2^{\text {nd }}$-order correlations. Neural networks with unsupervised learning rules are able to estimate the signal mixing to high accuracy, so that the original signals (independent components) can be recovered through inverse mixing.

The ability to blindly demix signals enables a novel form of security against those who may attack multimedia watermarks. One of the independent component signals in the mixed data stream could be a copy of an overt (visible or audible) authenticity mark on the host signal. A multimedia player enabled with an ICA neural network could then blindly demix the overt mark copy and determine whether the mark is still present in the host data.

If the overt authenticity mark has been removed, then a program triggers that takes appropriate action against the unauthorized data, e.g. degrading of quality. We call this program an electronic "bacterium," as opposed to a "virus," since it does not replicate indiscriminately. Moreover, there is a "vaccine" against this electronic bacterium, i.e., the presence of the authenticity mark. We also investigate two key requirements of the approach: (1) the robustness of ICA digital watermarking with respect to lossy compression and (2) the dynamic range of the digital watermark as clutter versus original music, for a signal coded under the MPEG-3.

\section{BLIND DEMIXING OF MUltimedia DATA WITH ICA NEU- RAL NETWORKS}

Existing watermarking systems are essentially non-blind, in the sense that they require original versions of both the host data and watermark keystream in order to extract the watermark. This may be reasonable for applications that merely verify the existence of the watermark for proving ownership. However, non-blind schemes are inadequate for our application, in which embedded data is extracted on the consumer side, not the producer side.

A straightforward way to combine host and watermark signals is to combine them linearly. The signals could then later be demixed given the linear mixing coefficients. However, this provides little in the way of flexibility or security. It would be much better if the signals could be demixed without knowledge of the original mixing coefficients, or even of the original signals themselves. Thus, we are faced with the problem of blind demixing of source signals.

In ICA, the source signals are modeled as statistically independent signal components, which have been subsequently mixed linearly. Independence is defined such that the joint probability densities of the signal components can be factorized as the product of the marginal densities. Thus, independence is over all orders of statistics, not just $2^{\text {nd }}$-order correlations.

Neural networks with unsupervised learning are able to estimate the signal mixing to high accuracy, so that the original signals (independent components) can be recovered through inverse mixing. The learning rules are based on maximizing the degree to which the independent components are nongaussian. Possible measures of nongaussianity are the absolute value of kurtosis ( $4^{\text {th }}$-order cumulant), or negentropy (slightly modified version of differential entropy).

ICA assumes that there are multiple sensors, each sensing a different mix of the independent components. Thus is extends conventional single-sensor processing of signals to multiple sensors, analogous to the multiple sensors (eyes and ears) in humans. The unsupervised ICA neural networks are able to simultaneously compare sensor outputs, extracting noise so that only coherent signals remain. In the unsupervised learning rule, there is no specific desired output other than white gaussian noise. This is consistent with the idea that what is not noise must be signals.

Fig. 3 is an example of the mixing of multimedia data, with blind demixing by an ICA unsupervised neural network. The three independent data components are the host data, an advertisement, and an authenticity mark. The data are mixed to insure the various components remain together for Internet distribution. The neural network with unsupervised learning estimates with high accuracy the mixing matrix $\mathbf{A}$, allowing blind demixing of the independent components.

\section{INDEPENDENT COMPONENTS FOR MULTIMEDIA WATERMARK VACCINATION}

It is important that ICA demixing be robust with respect to various forms of signal processing. Being linear, ICA demixing is invariant with respect to linear filtering. It is also invariant with respect to various changes in sampling, which cause synchronization problems with most other watermarking techniques. Such sampling changes include cropping, line dropping, or changing the sample rate. In the ICA model, each sample point is a mix of two independent components, and the mix is same for all samples.

It is also critical that ICA neural networks be able to demix signals that have been subjected to lossy compression. In general, compression can occur either before or after mixing. Compression before mixing yields nearly lossless demixing, and avoids costly decompression of previously compressed data. As we see in Fig. 4 for audio data, ICA neural networks can blindly demix signals with minimal distortion even after they have been subjected to the nonlinear transformation of lossy wavelet compression. 


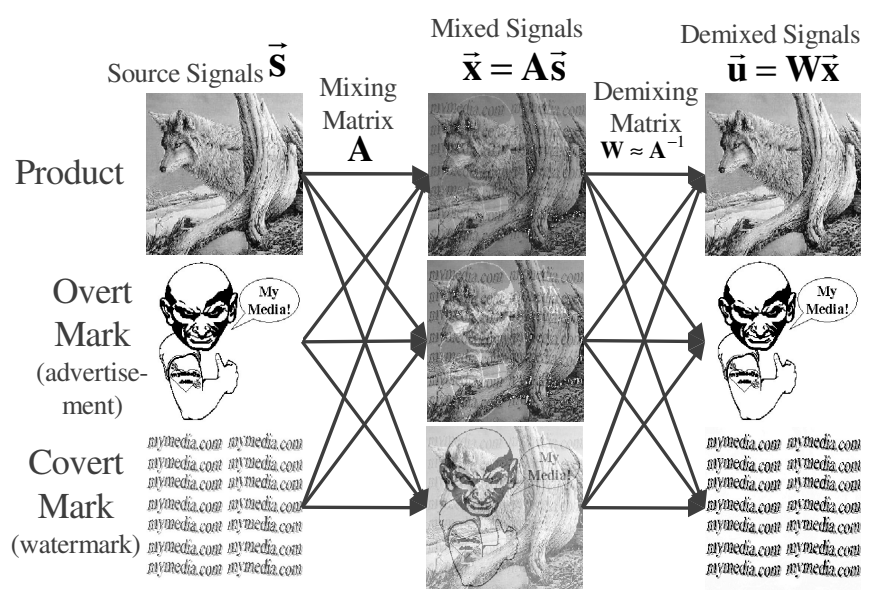

Fig. 3. Example mixing of multimedia data, with blind demixing via ICA unsupervised neural network.

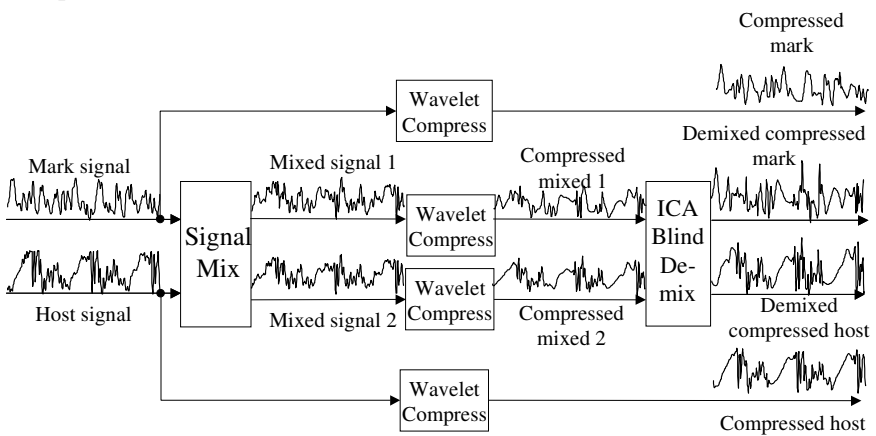

Fig.4. Robustness of ICA neural net blind demixing with respect to nonlinear wavelet audio data compression.

In Fig. 5, after the host and watermark images are mixed, the mixtures are subjected to DWT compression. ICA unsupervised neural nets are still able to blindly demix host and mark images, despite the nonlinear transformation of mixed images via compression. In fact, there is little visual difference compared to the compressed versions with no mixing involved.

Once the data is demixed, it is open to attack by the consumer. For example, the consumer could attempt to remove a visible overt company logo from the demixed multimedia product, to claim ownership. We do not have the luxury of a DVD-like approach, where there is industry-wide cooperation and all multimedia players are trusted. Our approach needs to be completely autonomous.

Our approach to making product authenticity secure against someone who has the demix key is essentially a watermark "vaccination" against digital "bacteria." The dormant bacterium is released at Internet download time, along with the mixed multimedia product. It is then made active when the product is demixed. The purpose of the bacteria is to "infect" the multimedia product if it is tampered with, e.g. its company $\log$ is removed. It is localized completely to the product it protects.

The vaccination is the actual presence of the overt watermark, and it has already been administered in the sense that
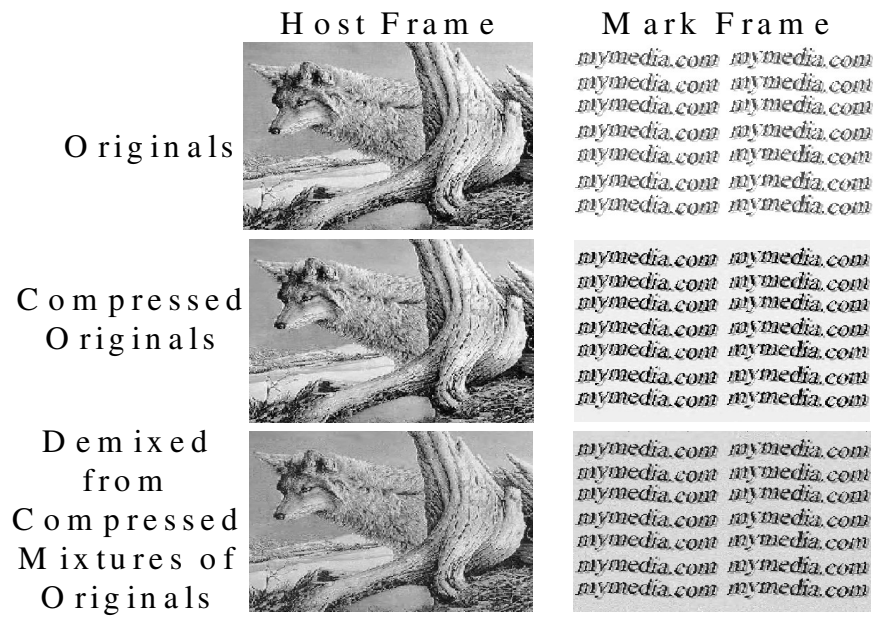

Fig.5. Robustness of ICA neural net blind demixing with respect to nonlinear wavelet image data compression.

the watermark is initially part of the product. The level of infection, that is, the action to take upon logo deletion, can vary from doing nothing to rendering the multimedia product unusable. This is according to the choice of product owner, as consistent with the owner's philosophy and goals. The infection is localized to the unauthorized multimedia file, and spreads to no other part of computer, since unauthorized content is directly recognizable via the covert watermark.

The computer virus ${ }^{1}$ was first described by Cohen in 1984 [15]. For various reasons, computer viruses are perceived to be malicious code with no useful purpose. However, maliciousness is neither a necessary nor a sufficient property for computer viruses. In fact, Cohen described the potential advantages of beneficial viruses.

While so far there have been relatively few successful examples of beneficial viruses, the failed attempts generally violate one or more important properties [16]. In particular, beneficial virus-like programs should be controllable, recognizable, waste few resources, have a bug-containment mechanism, be compatible with other software components, and be more effective than non-virus alternatives.

We follow a model for beneficial virus-like programs [16] that satisfies these requirements. It requires user consent via cryptographically strong system authentication. That is, the digital bacteria only spread to machines that have agreed to the network policy for unauthorized media. These digital bacteria are strictly controlled via public-key cryptography for authentication. As we describe in [17], our digital bacteria meet the established requirements for beneficial virus-like programs.

One way to implement watermark vaccination is to have the vaccination-checking executable code downloaded along

\footnotetext{
${ }^{1}$ In the early 1980s, as a Ph.D. student at the University of Southern California, Fred Cohen got the idea of self-replicating software that spreads by attaching itself to existing programs. He shared this idea with his thesis advisor Len Adleman, who pointed out the similarity to a biological virus, leading to the term "computer virus."
} 


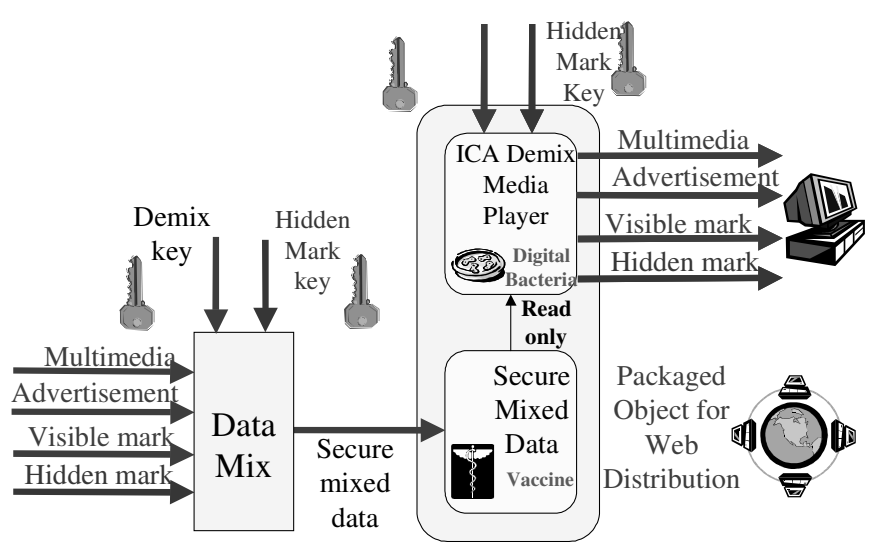

Fig. 6. Object-based model for multimedia product protection by watermark vaccination.

with the mixed data and multimedia player. It is then executed along with the player, as shown in Fig. 8. Multimedia data, advertisement, and mark data are mixed and secured, then embedded within the object. The neural network ICA demix media player is defined as an object method, with read-only access to mixed data. The demix key allows data to be demixed, and the mark key allows the hidden mark to be accessed for copyright proof.

Another implementation is DVD-like, that is, via trusted players. Either the multimedia producers or a third party provides the player/protection software cost-free. This approach requires universal participation for complete protection; otherwise, there is protection only among trusted players. A third approach is to implement a DVD-like scheme in hardware, with an interface to the operating system.

\section{EXPERIMENTAL BACKGROUND}

\section{A. Blind Demixing with ICA}

The idea is to linearly mix a watermark acoustic signal with the music signal, MP3 encode and decode the resulting signal, then attempt to demix the watermark signal from the music. The novelty of this method introduces difficulty in the removal of the watermark by the unsophisticated consumer. The value of restricted computer codes to restore the music from a watermarked signal will depend in part on the quality of music after blind demixing with ICA [18]-[23]. We measure the watermark contamination of the music after blind demixing with ICA with MP3 encoding. The receiver noise is negligible relative to the source signal power.

It has been shown [18][19] that maximization of the joint entropy of the output $H(y)$ (equivalently minimization of the mutual information between the output components $I(\boldsymbol{y}))$ by computation of the gradient with respect to the weight matrix $\boldsymbol{W}$.

\section{B. MP3 Encoding/Decoding}

The compact MP3 encoding format is a lossy compression method. It utilizes a psychoacoustic model of human hearing to discard information [24][25]. Human hearing is subject to

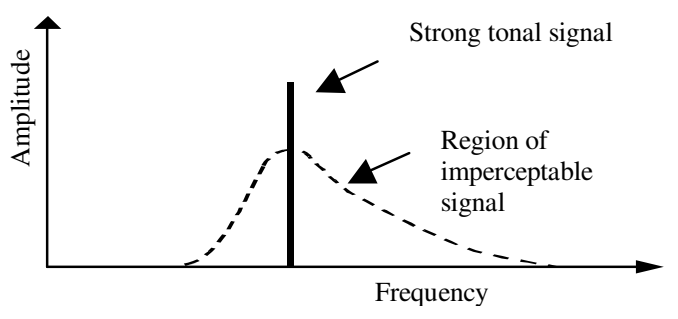

Fig. 7. Illustration of audio noise masking. Weak signals in the local frequency neighborhood of a strong tone are imperceptible [24].

auditory masking, where small tones in the local frequency neighborhood of a much stronger tone are imperceptible (see Fig. 9). Further, the human auditory system's frequency resolution is a function of absolute frequency; perceptible filter widths are narrow at the low end and significantly wider at the high end. Thus, weak tones adjacent to stronger tones may be eliminated by MP3 encoding/decoding.

Several issues arise when considering ICA for blind demixing of MP3 watermarked music. First, introduction of a watermark signal must be considered in the context of the music, so that it will not be eliminated by the lossy MP3 encoding/decoding. A second issue is consideration of the statistics of the music and watermark signals after MP3 encoding/decoding. The concern is that the statistics of both the watermark and music signals may be perturbed when selected frequency components are nulled. The altered statistics may adversely affect the ICA blind demixing.

Our strategy is to measure the watermark contamination in the music signal after blind demixing with ICA. First we measure the watermark residue in the music channel without MP3 encoding/decoding. Then the measurement is repeated after MP3 encoding/decoding. The next section discusses the methodology and equipment configuration for our measurements.

\section{METHODOLOGY}

The goal is to measure the watermark contamination in the music signal after blind demixing with ICA. First a baseline is produced that measures the watermark residue in the music channel when the MP3 codec is not employed, in Fig. 10 without the dashed block. Then the residue level is measured by introducing the MP3 codec to the linearly mixed signals as in Fig. 10 with the dashed block. To control the relative contributions of the watermark and music signals, the linear mixing matrix was parameterized for a single mixture angle $\boldsymbol{\theta}$. In both cases, the latter are demixed using ICA to recover permuted and scaled versions of the originals (Music' and Watermark').

To facilitate simultaneous linearity and residue measurements, the watermark signal and music signals were implemented as a pair of dual tones. The Watermark signal is two equal amplitude tones at $900 \mathrm{~Hz}$ and $1000 \mathrm{~Hz}$ and two equal amplitude tones for the Music signal are at $2100 \mathrm{~Hz}$ and 2250 $\mathrm{Hz}$. The linear dynamic range is determined by measuring the magnitude of the intermodulation (IM) products generated by 


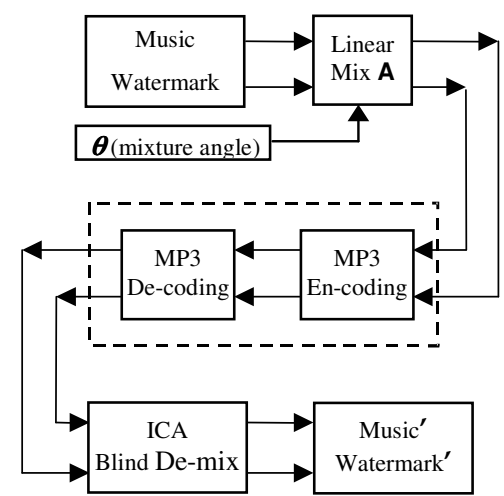

Fig. 8. Mixing and demixing procedure model with/without MP3 codec block (dashed block).

the tone pair, relative to the magnitude of the respective tone pairs. As an example, for the two tone watermark signal at 900 and $1000 \mathrm{~Hz}$, any $1^{\text {st }}$ order IM products would occur at 800 and $1100 \mathrm{~Hz}$.

The MP3 codec was implemented using commercial software [26]. The following MP3 encoding options were chosen and held constant through all the experiments: $44.1 \mathrm{kHz}$ sample rate, $128 \mathrm{kbps}$ per channel, and stereo (no joint stereo coding).

As a check, the software MP3 decoder was checked with consumer hardware. MP3 files were burned on CD-ROMs, played back on a Riovolt MP3/CD Player through an RCA SA-155 Integrated Stereo Amplifier (Radio Shack catalog \#31-5000) with spectrum measurements obtained from a spectrum analyzer (HP 3588A with opt. 001). The nominal $80 \mathrm{~dB}$ of linear dynamic range measured in the MP3 software codec was also observed with the hardware.

\section{RESULTS}

An illustration of the results of demixing for a mixture angle of 30 degrees in the absence of the MP3 codec is shown in Fig. 11. It shows more than $80 \mathrm{~dB}$ of isolation between the wanted signals and the residue signals. The left column is Watermark' channel with the wanted watermark tones at 900 $\mathrm{Hz}$ and $1000 \mathrm{~Hz}$, and the right column is Music' channel with the wanted music tones at $2100 \mathrm{~Hz}$ and $2250 \mathrm{~Hz}$.

Fig. 12 is the summary of minimum magnitude differences in $\mathrm{dB}$ for the case when the MP3 codec (the dashed block in Fig. 10) was absent and the mixture angle was varied from 0 to 90 degrees at 0.1 -degree increments in the top of pictures. In the bottom of pictures of Fig. 12 is a summary for the case when the MP3 codec (the dashed block in Fig. 10) was present, but the residues were evaluated only from 0 degree to 90 degree at every 1 degree increments. In this figure, the left column shows the minimal magnitude differences in $\mathrm{dB}$ between the residue signal and the wanted signal in Watermark' channel, and in the same way the right column shows the minimal magnitude differences in Music' channel.
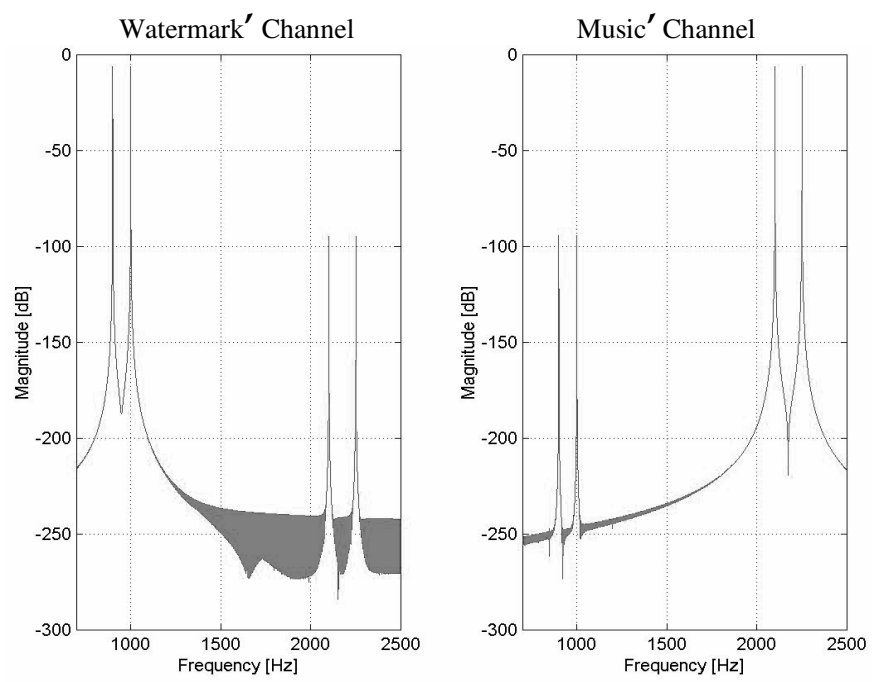

Fig. 9. The residues in both demixed channels.
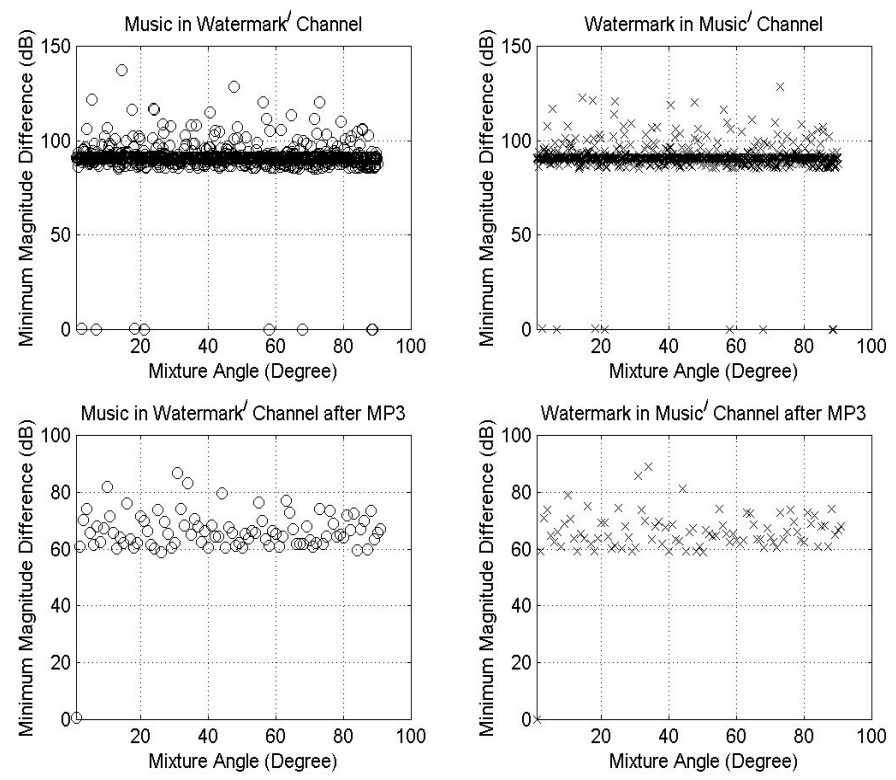

Fig. 10. Minimum magnitude differences $(\mathrm{dB})$ between the residue signal and the wanted signal are shown at each mixture angle from 0 to 90 degrees.

\section{DISCUSSION AND CONCLUSIONS}

This paper examines the application of independent component analysis (ICA), via unsupervised neural networks, to authenticity protection for multimedia products. The blind demixing capability of these neural networks extends signal processing from a one-sensor approach to a multi-sensor approach.

For watermark security, we propose a covert watermarking signal that serves as a vaccination against a dormant digital bacterium. Removal of the watermark triggers the bacterium, which takes the appropriate action against the pirated product. Our digital bacteria meet established requirements for beneficial virus-like programs. 
We introduce a novel method for watermarking digitally encoded acoustic music files. A watermarked signal is linearly mixed with a music signal. The resulting mixture pair is put through an MP3 codec, and then blindly demixed with ICA to recover the music signal. The watermark contamination in the music channel is measured for the two cases: when the MP3 codec was absent and when it was present.

Without the MP3 codec, the results of blind demixing with ICA show watermark attenuation in the music channel is more than $82 \mathrm{~dB}$ for a wide range of mixture angles, close to the $90 \mathrm{~dB}$ dynamic range of MP3 encoded music. When the linearly mixed signals are passed through the lossy MP3 co$\mathrm{dec}$, the residues are approximately $70 \mathrm{~dB}$ down from the wanted signals as measured at the 30 and 60 degrees mixture angles. However, when we measure from 0 to 90 degrees at every degree, the residues are approximately larger than 59 $\mathrm{dB}$ down. Despite the $23 \mathrm{~dB}$ performance deficiencies with the MP3 codec, the watermark contamination in the music channel is likely to be imperceptible by the human auditory system. The high quality of the music recovery suggests that there is value in restricted computer codes for the restoration of music from watermarked MP3 digital music files.

The lossy MP3 coding format might affect the statistics of both the watermark and music signals when selected frequency components are nulled. However, in our evaluations with the selected simple tonal pairs representing the watermark and acoustic signals, the adverse affects on blind demixing with ICA are small.

Some open research opportunities include recovery of the absolute amplitude and phase of the music signal. With ICA blind demixing an unknown scale and permutation in the recovered outputs generally occurs. A scale change could also manifest itself as a 180-degree phase shift in the music channel. For stereo music, this may destroy the "depth" perception. If some of the properties of the watermark are known a priori, then recovery of the watermark might serve as a guide to recover the music channel's absolute amplitude and sense of phase.

In summary, our experimental results show that watermark contamination in the music channel is likely to be inaudible, and that the adverse affects on blind demixing with ICA are small. We conclude that our approach can provide a flexible, robust, and secure system for protecting the authenticity of multimedia products.

\section{REFERENCES}

[1] S. Noel and H. Szu, "Multimedia authenticity with ICA watermarks," Wavelet Applications VII, SPIE Proc., Vol. 4056, pp. 175-184, April 26-28, 2000.

[2] H. Szu, "Progress in unsupervised artificial neural networks for image demixing applications," IEEE Industrial Electronics Society Newsletter, Vol. 46, No. 2, pp. 7-12, June 1999.

[3] H. Szu, "ICA-an enabling tech for intelligent sensory processing," IEEE Circuits and Systems Newsletters, pp. 14-41, December 1999.

[4] A. X. Quan, H. H. Szu, and Z. Markovitz, "Local ICA for the most wanted face recognition," Wavelet Applications VII, SPIE Proc., Vol. 4056, pp. 539-551, April 26-28, 2000.
[5] F. Hartung and M. Kutter, "Multimedia watermarking techniques," Proceedings of the IEEE, Vol. 87, No. 7, pp. 1079-1107, July 1999.

[6] M. Swanson, M. Kobayashi, and A. Tewfick, "Multimedia dataembedding and watermarking technologies," Proceedings of the IEEE, Vol. 86, No. 6, June 1998.

[7] P. Jessop, "The business case for audio watermarking," IEEE International Conference on Acoustics, Speech, and Signal Processing, pp. 2077-2074, 1999.

[8] I. Kopriva and H. Szu, "Blind inversion in nonlinear space-variant imaging by using cauchy machine," SPIE Proceedings, Vol. 5102 Independent Component Analyses, Neural Nets and Wavelets, Orlando, FL, April 21-25, 2003.

[9] S.-B. Yim, J. Willey, J. Landa, and H. Szu, "Watermarking MP3 encoded music and ICA blind de-mixing," SPIE Proceedings, Vol. 5102 Independent Component Analyses, Neural Nets and Wavelets, Orlando, FL, April 21-25, 2003.

[10] R. Barnett, "Digital watermarking: applications, techniques, and challenges," Electronics and Communication Engineering Journal, Vol. 11, No. 4, pp. 173-183, August 1999.

[11] J. W. Seok and J. W. Hong, "Audio watermarking for copyright protection of digital audio data," Electronics Letters, Vol. 37, No. 1, pp.60-61, $4^{\text {th }}$ January 2001.

[12] D. Yu, F. Satter, and Kai-Kuang Ma, "Watermark detection and extraction using ICA method," EURASIP Journal on Applied Signal Processing, Vol. 2002, No. 1, pp. 92-104, January 2002.

[13] D. Yu and F. Satter, "Robust image watermarking based on independent component analysis," 2002 IEEE International Conference on Acoustics, Speech, and Signal Processing, Vol. 4, pp. 4177-4177, 2002.

[14] F. J. Gonzalez-Serrano, H. Y. Molina-Bulla, and J. J. Murillo-Fuentes, "Independent component analysis applied to digital image watermarking," ICASSP 2001: Proceedings of IEEE International Conference on Acoustics, Speech, and Signal Processing, Vol. 3, pp. 1997-2000, Salt Lake City, UT, May 2001.

[15] F. Cohen, "Computer viruses - theory and experiments," originally appearing in IFIP-sec 84, also appearing in DOD/NBS 7th Conference on Computer Security, and IFIP-TC11 Computers and Security, Vol. 6, 1987.

[16] V. Bontchev, "Are 'good' computer viruses still a bad idea?," Proceedings of EICAR, London, 1994.

[17] H. Szu, S. Noel, S.-B. Yim, J. Willey, and J. Landa, "Multimedia authenticity protection with ICA watermarking and digital bacteria vaccination," Neural Networks, special issue for International Joint Conference on Neural Networks, 2003.

[18] A. J. Bell and T. J. Sejnowski, "An information-maximization approach to blind separation and blind deconvolution," Neural Computation, Vol. 7, pp. 1129-1159, 1995.

[19] A. J. Bell and T. J. Sejnowski, "Learning the higher-order structure of a natural sound," Network: Computation in Neural Systems, Vol. 7, pp. 261-266, 1996.

[20] S. Amari, A. Chichocki, and H. Yang, "A new learning algorithm for blind signal separation," In Advance in Neural Information Processing Systems, Vol. 8, pp. 757-763, 1996.

[21] E. Oja and J. Karhunen, "Signal sepration by nonlinear Hebbian learning," Proceedings of ICNN-95, pp.417-421, Perth, Australia, 1995.

[22] P. Comon, "Independent component analysis, a new concept?," Signal Processing, Vol. 36, No. 3, pp. 287-314, April 1994.

[23] A. Hyvärinen and E. Oja, "Independent component analysis: a tutorial," tutorial notes for International Joint Conference on Neural Networks (IJCNN' 99), Washington, DC, [http://www.cis.hut.fi/project/ ica/], July 1999.

[24] D. Pan, "A tutorial on MPEG/audio compression," IEEE Multimedia Journal, pp. 60-74, Summer 1995.

[25] K. Brandenburg and H. Popp, "An introduction to MPEG Layer-3," EBU Technical Review, available at [http://www.ebu.ch/trev_283popp.pdf], June 2000.

[26] MP3 codec implemented with CoolEdit 2000 version 1.1 Build 2418, software from Syntrillium Software Corporation, P.O. Box 62255, Phoenix, AZ. 